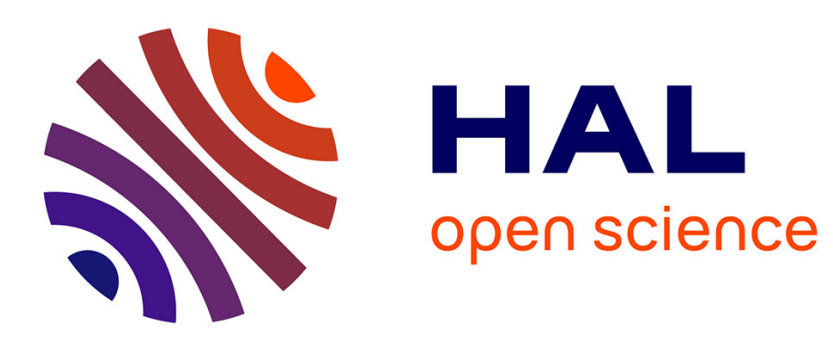

\title{
Pricing of differentiated-QoS services WiMAX networks
}

\author{
Aymen Belghith, Loutfi Nuaymi, Patrick Maillé
}

\section{To cite this version:}

Aymen Belghith, Loutfi Nuaymi, Patrick Maillé. Pricing of differentiated-QoS services WiMAX networks. Globecom'08: IEEE Global Communication Conference, 30 November - 4 December, New Orleans, LA, USA, Nov 2008, New Orleans, United States. 10.1109/GLOCOM.2008.ECP.990 . hal02120775

\section{HAL Id: hal-02120775 \\ https://hal.science/hal-02120775}

Submitted on 6 May 2019

HAL is a multi-disciplinary open access archive for the deposit and dissemination of scientific research documents, whether they are published or not. The documents may come from teaching and research institutions in France or abroad, or from public or private research centers.
L'archive ouverte pluridisciplinaire HAL, est destinée au dépôt et à la diffusion de documents scientifiques de niveau recherche, publiés ou non, émanant des établissements d'enseignement et de recherche français ou étrangers, des laboratoires publics ou privés. 


\title{
Pricing of differentiated-QoS services WiMAX networks
}

\author{
Aymen Belghith*, Loutfi Nuaymi* and Patrick Maille* \\ *TELECOM Bretagne, France \\ 2 rue de la châtaigneraie, CS 17607, 35576 \\ Email: \{first.last $\} @$ telecom-bretagne.eu
}

\begin{abstract}
WiMAX/IEEE 802.16 system is a very open Broadband Wireless Access (BWA) technology with different possible services. Pricing of WiMAX is an important topic with different optimization possibilities. In a previous paper, we investigated WiMAX pricing for real-time applications (Unsolicited Grant Service (UGS) and real-time Polling Service (rtPS) QoS classes of WiMAX) [7]. We add the Best Effort (BE) QOS class in this paper in order to have a rather complete WiMAX environment. The system model is described. Then we consider two different BE pricing mechanisms: fixed price and auction-based variable price. The proposed methods are evaluated through simulations in order to have some interesting comments and highlights for WiMAX pricing.
\end{abstract}

\section{INTRODUCTION}

WiMAX is a powerful Broadband Wireless Access (BWA) technology. It is based on the IEEE 802.16 standard [1] and its amendment [2]. WiMAX has a very rich set of features in order to have a highly-efficient use of the radio resource while transmitting different service types which allows different constraints.

The IEEE 802.16 BWA system has three possible physical (PHY) layers: Single Carrier (SC), Orthogonal Frequency Division Multiplexing (OFDM), and Orthogonal Frequency Division Multiple Access (OFDMA). WiMAX retains only OFDM and OFDMA. Both Duplexing modes are possible: Time Division Duplexing (TDD) and Frequency Division Duplexing (FDD). For topologies, Point-to-MultiPoint (PMP) and Mesh modes can be used. We consider the PMP transmission mode and the FDD duplex mode. Our study could be extended to the TDD mode, with additional latitude.

The IEEE 802.16 standard Medium Access Control (MAC) layer provides QoS differentiation for the different types of applications that might operate over 802.16 networks, through the defined Scheduling Service Types also called QoS Classes. There are five QoS classes in IEEE 802.16. Four classes of QoS were defined in 802.16-2004 standard : Unsolicited Grant Service (UGS), real-time Polling Service (rtPS), nonreal-time Polling Service (nrtPS), and Best Effort (BE). A fifth one has been added with 802.16e amendment: extended real-time Polling Service (ertPS) class. The MAC Convergence Sublayer (CS) operates the classification of the different users applications in those five classes.

For these different services transmitted in a quickly varying wireless environment, the pricing strategy is an open problem, actually an important and challenging one. Pricing models are defined for the integration of WiMAX and Wireless Fidelity (WiFi). A strategy game used in economics, called Stackelberg leadership model, is used in [3], in order to propose a pricing model for sharing bandwidth between WiMAX and WiFi users. Other pricing models are defined only for WiMAX context. Two market models are defined in [4]. The Base Station (BS) allocates resources only for the residential users in the first market model. In the second model, the BS also provides services to the Small and Medium Enterprises (SME) customers. In [5], a pricing model for wireless networks users is proposed. The utility function of the proposed pricing model depends on the transmission rate and the quality of the channel. In [6], a cost-based Call Admission Control (CAC) is proposed for IEEE 802.16 WiMAX. It is based on the required bandwidth and the residual bandwidth. The competitive OnLine (COL) is used in order to have an exponential cost function of the residential bandwidth. Since WiMAX operators can use different pricing strategies for the QoS classes, we propose pricing schemes for two WiMAX QoS classes in [7]. Our pricing model is designed for real-time applications.

In this paper, we propose two different pricing schemes for the BE QoS class in an environment where the UGS and rtPS QoS classes are also considered.

This paper is organized as follows. In Section II, we present the system model. Then, we describe our pricing proposals for UGS, rtPS, and BE QoS classes. In Section III, we present some simulation results for our two proposed BE pricing mechanisms. Conclusions and directions for future work are provided in Section IV.

\section{SYSTEM MODEL}

\section{A. WiMAX model}

We consider a simplified environment with only three QoS classes of WiMAX services (out of the five standard-defined classes):

- UGS: supports real-time service flows that have fixed-size data packets on a periodic basis designed for circuit-type voice services, for instance.

- rtPS: supports real-time service flows that generate variable-size data packets on a periodic basis (ex: video services). A minimum transmission rate $\left(R_{m i n}\right)$ is guaranteed, and the rate can be increased up to a maximum transmission rate $\left(R_{\max }\right)$. 
TABLE I

NUMBER OF USEFUL BITS PER OFDM SYMBOL

\begin{tabular}{|c|c|c|c|}
\hline Modulation & Coding & Receiver SNR (dB) & $\begin{array}{l}\text { Number of useful bits } \\
\text { per OFDM symbol }\end{array}$ \\
\hline \hline BPSK & $1 / 2$ & 3.0 & $192 \times 1 \times 1 / 2=96$ \\
\hline \multirow{2}{*}{ QPSK } & $1 / 2$ & 6.0 & $192 \times 2 \times 1 / 2=192$ \\
\cline { 2 - 4 } & $3 / 4$ & 8.5 & $192 \times 2 \times 2 / 3=288$ \\
\hline \multirow{2}{*}{ 6-QAM } & $1 / 2$ & 11.5 & $192 \times 4 \times 1 / 2=384$ \\
\cline { 2 - 4 } & $3 / 4$ & 15.0 & $192 \times 4 \times 2 / 3=576$ \\
\hline \multirow{2}{*}{ 64-QAM } & $2 / 3$ & 19.0 & $192 \times 6 \times 2 / 3=768$ \\
\cline { 2 - 4 } & $3 / 4$ & 21.0 & $192 \times 6 \times 3 / 4=864$ \\
\hline
\end{tabular}

- BE: designed for non-real-time applications where no guarantee is provided. This class should evidently be the cheapest one.

An objective of a pricing scheme could be to maximize the network revenue, while still satisfying some fairness constraints. Pricing schemes objectives and equity constraints are studied in this paper. We are moreover looking for a scheme that remains simple to implement and to understand for users.

Different frame duration and bandwidth values can be used in IEEE 802.16. For a given system, the frame has a fixed number of OFDM symbols $\left(N_{O F D M}\right)$ when the OFDM PHY layer is considered. The number of symbols depends on some parameters such as the frequency bandwidth and the Cyclic Prefix (CP). An appropriately-designed pricing mechanism should then properly allocate those symbols among users, so as to satisfy some QoS agreements, or maximize user satisfaction or provider revenue. The number of useful data bits is indeed variable and depends on the Modulation and Coding Scheme (MCS) used by the considered user. The MCS to be used is defined by the link adaptation procedure. The choice of the appropriate MCS depends on the value of receiver Signalto-Noise Ratio (SNR). The transmitting station (BS or SS) switches to a more energy-efficient MCS if the SNR is good. It switches to a more robust MCS if the SNR is poor. Once the MCS is defined, the number of bits per OFDM symbol, and then the useful number of bits per frame, is known as shown in Table I. The BPSK modulation is mainly used for management purposes and will not be considered in this work. Therefore, the BS must take into account link adaptation in its scheduling considerations. The link adaptation process must also be taken into account in the pricing scheme.

\section{B. Pricing Model}

We propose a utility function for each type of service. The utility is the difference between the price that the user is willing to pay for the service (that we call valuation or willingness to pay) and the price actually paid. The utility, valuation, and price actually paid are per unit of time.

$$
U(r)=V(r)-P(r)
$$

where:

- $U$ : represents the utility function.

- $V$ : represents the valuation function.

- $P$ : represents the price actually paid function.
- $r$ : represents the throughput of the connection (expressed in $\mathrm{kbit} / \mathrm{s})$.

1) UGS users: The transmission rate of the UGS connections is fixed at the beginning of the session. We consider that each UGS user $i$ is willing to pay a given price $v_{i}$ per transmitted kbit per second.

$$
V_{U G S}(r)=v_{i} \cdot r
$$

We suggest to use a pricing scheme based on two prices.

$$
P_{U G S}(r)= \begin{cases}P_{U G S_{-} L o w} \cdot r, & \text { if } \frac{n b_{r e s}}{n b_{t o t}} \leq t h_{U G S} \\ P_{U G S_{-} H i g h} \cdot r, & \text { otherwise }\end{cases}
$$

where:

- $P_{U G S_{-} L o w}:$ represents the price charged when the network is not congested.

- $P_{U G S_{-} H i g h}$ : represents the price charged during the congestion periods. We consider that $P_{U G S_{-} H i g h}=\alpha_{U G S}$. $P_{U G S_{-} L o w}$.

- $n b_{r e s}$ : represents the number symbols already reserved for the UGS connections.

- $n b_{t o t}$ : represents the total number of symbols per frame.

- $t h_{U G S}$ : represents a threshold that determines when the network is congested.

2) $r t P S$ users: rtPS services offer a flexibility in the transmission rate. We therefore consider that users have elastic demand, i.e. their valuation function is increasing and concave in the transmission rate $r$.

$$
V_{r t P S}(r)=\left\{\begin{array}{l}
v_{i} \cdot\left(\frac{-r^{2}}{R_{i \_} \max }+2 r\right), \quad \text { if } r \leq R_{i \_} \max \\
v_{i} \cdot R_{i_{-} \max }, \quad \text { otherwise }
\end{array}\right.
$$

where:

- $r$ : represents the reserved rate.

- $v_{i}$ : represents a constant coefficient (in monetary unit (MU) by kbit/s transmitted).

- $R_{i \_ \text {max }}$ : represents the maximum transmission rate of the connection $i$.

An rtPS user $i$ with transmission rate $r$ that belongs to $\left[R_{\min }, R_{\max }\right]$ is charged a price:

$P_{r t P S}(r)=P_{r t P S \_m i n} \cdot R_{m i n}+P_{r t P S \_a d d} \cdot\left(r-R_{m i n}\right)$

where:

- $P_{r t P S_{-} m i n}$ : represents a fixed unit price for $R_{\min }$. It is independent of the congestion.

- $P_{r t P S \_a d d}:$ represents a variable unit price for the additional transmission rate. It increases with congestion.

The price for extra rate is chosen as:

$$
P_{r t P S \_a d d}=\frac{C_{r t P S}}{n b_{r e m \_m i n}}
$$

where:

- $C_{r t P S}$ : represents a pricing constant fixed by the operator.

- $n b_{\text {rem_min }}$ : represents the number of OFDM symbols remaining after all the UGS users are served and the rtPS users get their minimum transmission rate. 
3) BE users: The BE connections have no QoS guarantees. Therefore, the BE users have flexibility in their transmission rate. We consider that their valuation function is increasing and concave in the number of reserved symbols $s$. We propose:

$$
V_{B E}(s)=\left\{\begin{array}{l}
\gamma_{i} \cdot\left(\frac{-s^{2}}{S_{\max }}+2 s\right), \quad \text { if } s \leq S_{\max } \\
\gamma_{i} \cdot S_{\max }, \quad \text { otherwise }
\end{array}\right.
$$

where:

- $s$ : represents the number of reserved symbols.

- $\gamma_{i}$ : represents a constant coefficient (in MU by reserved symbol).

- $S_{\max }$ : represents the number of reserved symbols above which the valuation of $\mathrm{BE}$ users remains constant.

We also introduce heterogeneity among the BE users by assuming that $\gamma_{i}$ is randomly chosen for each user, according to continuous uniform distribution on the interval $\left[\Gamma 1_{B E}\right.$, $\left.\Gamma 2_{B E}\right]$ (in MU by reserved symbol).

The BE calls are charged a price SymbolPrice $B E$ per reserved symbol.

$$
P_{B E}(s)=\text { Symbol_Price }{ }_{B E} \cdot s
$$

We then suggest to use two pricing schemes. In the first pricing model, called Fixed Symbol Price Model (FSPM), the operator charges a fixed price SymbolPrice $B E$ per reserved symbol. After serving the UGS and rtPS users, the remaining symbols are equitably distributed between all the BE users, using the Round Robin (RR) scheduler. The number of reserved symbols to each connection also depends on the utility function and the the remaining size of the file to be transmitted.

In the second pricing model, called Variable Symbol Price Model (VSPM), the operator charges, using also (8), a variable price Symbol_Price $B E$ per reserved symbol.

As a specific variable prices, we suggest to allocate the OFDM symbols that are not used by UGS or rtPS calls using price auctions, i.e. with a pricing scheme that gives symbols to users who value them most. A simple way to obtain such an allocation is to determine the market clearing price [8], that is the unit selling price for which the demand of all BE users equals the number of available OFDM symbols. We assume that such an ideal scheme is used. We can imagine auction or tâtonnement methods to converge to this price [9]. The definition of the practical details of the auction mechanism in WiMAX system can be an interesting future topic of research.

The main objective of VSPM is to find the maximum reserved symbol price while reserving all the remaining symbols. From (7), the number of symbols to reserve for each connection $i$, noted $d_{i}(p)$, is expressed as follows.

$$
d_{i}(p)=\left(V_{i}^{\prime}\right)^{-1}(p)=S_{\max } \cdot\left[1-\frac{p}{2 \gamma_{i}}\right]^{+}
$$

Therefore, the value of Symbol_Price ${ }_{B E}$ is determined as the solution $p$ of the equation:.

$$
\sum_{i=1}^{N u s e r_{-} B E} S_{\max } \cdot\left[1-\frac{p}{2 \gamma_{i}}\right]^{+}=n b_{B E}
$$

where:

- Nuser ${ }_{B E}$ : represents the number of the BE users.

- $p$ : represents the price of a reserved OFDM symbol.

- $n b_{B E}$ represents the number of remaining symbols to allocate to the BE users.

If $N u s e r_{-} B E \leq \frac{n b_{B E}}{S_{\max }}$, the solution of (10) is negative. In this case, there is no congestion and then we can offer a free access to the network $(p=0)$.

\section{Simulation Results}

\section{A. Simulation model}

This section describes the situation we have considered to simulate the behavior of a WiMAX system under the pricing schemes described in the previous section. In this work, we consider the OFDM PHY layer used in the PMP topology and FDD mode. The number of OFDM symbols per frame $\left(N_{O F D M}\right)$ is constant and depends on the frame duration and the OFDM symbol duration. According to [10], the OFDM symbol duration is computed as follows:

$$
\text { OFDM symbol Duration }=\frac{1+G}{n \cdot \frac{B W}{N_{F T T}}}
$$

where:

- $G$ : represents the ratio of the guard time to the useful symbol time.

- $n$ : represents the the sampling factor.

- $B W$ : represents the channel bandwidth.

- $N_{F T T}$ : represents the total number of subcarriers. For the OFDM PHY, the total number of subcarriers is equal to $256\left(N_{F T T}=256\right)$.

For the following parameter values: $B W=7 \mathrm{MHz}, n=8 / 7$, $G=1 / 8$, and frame duration $=10 \mathrm{~ms}$ obtain an OFDM symbol duration equal to $35.86 \mu$ s and $N_{O F D M}$ equal to 278 symbols. The simulation duration is equal to $300 \mathrm{~s}$.

In our simulation framework, we consider Poisson arrivals of each class of customer UGS and rtPS, with respective arrival rate $\lambda_{U G S}=\lambda_{r t P S}=\lambda_{B E}=1 / 30 \mathrm{~s}^{-1}$. We assume that the durations of the UGS and rtPS sessions follow exponential distributions with respective mean $\mu_{U G S}=\mu_{r t P S}=5$ minutes. $\mathrm{BE}$ sessions correspond to file transfers and their duration therefore depends on the transmission rate experienced. We assume that the size of files to be transmitted by BE sessions follows a Normal law with mean $300 \mathrm{kbit}$ and standard deviation 150 kbit (eventually removing negative values).

We consider three types of UGS users: $10 \mathrm{kbit} / \mathrm{s}, 50 \mathrm{kbit} / \mathrm{s}$, and $200 \mathrm{kbit} / \mathrm{s}$, that represent $25 \%, 25 \%$, and $50 \%$ of the UGS users, respectively. We also consider four types of rtPS users, with respective $R_{\min }=30,50,100,200$ (in kbit/s) and $R_{\max }$ $=100,300,500,800$ (in kbit/s). We assume that each of these $\left[R_{\min }, R_{\max }\right]$ couples is chosen by $25 \%$ of the rtPS users.

The user SNR values are randomly chosen at the beginning of the session, considering a simple cellular model with three 
TABLE II

DISTRIBUTION OF THE MCSS FOR ARRIVING USERS

\begin{tabular}{|c|c|}
\hline SNR range $(\mathrm{dB})$ & Proportion of users \\
\hline \hline$[6,8.5)$ & $30 \%$ \\
\hline$[8.5,11.5)$ & $10 \%$ \\
\hline$[11.5,15)$ & $20 \%$ \\
\hline$[15,19)$ & $15 \%$ \\
\hline$[19,21)$ & $5 \%$ \\
\hline$[21,+\infty)$ & $20 \%$ \\
\hline
\end{tabular}

cells per cluster: the SNR distribution can then be obtained using classical results of the SNR repartition in cellular networks [11]. Having the SNR values, the MCS used is directly deduced from Table I. The distribution of SNRs (and then MCSs) is given in Table II.

The valuation parameter $\gamma_{i}$ (in MU by symbol reserved) of the BE users uniformly distributed on the interval $\left[\Gamma 1_{B E}\right.$, $\left.\Gamma 2_{B E}\right]$. We assume that $\Gamma 1_{B E}=0.05$ and $\Gamma 2_{B E}=0.5$.

Since the UGS and rtPS QoS classes are designed for realtime applications, an admission control mechanism is required. An UGS (resp. rtPS) customer is admitted only if there are enough OFDM symbols, taking into account its MCS used, for its data rate (resp. for its minimum data rate $R_{\min }$ ).

In this paper, we assume that $P_{U G S \_L o w}=P_{r t P S \_ \text {min }}=$ $P_{\text {ref }}=0.25$. For the UGS pricing model, we adopt $\bar{\alpha}_{U G S}=$ $2\left(P_{U G S \_H i g h}=2 * P_{\text {ref }}\right), t h_{U G S}=0.5$. For the rtPS pricing model, we adopt $C_{r t P S}=10$. The model details of the UGS and rtPS connections are studied in [7]. In this Section, we only focus on our two pricing models for the BE users.

\section{B. Investigation of $S_{\max }$}

Recall that $S_{\max }$ represents the number of reserved symbols above which the valuation remains constant (see (7)). Symbol_Price $_{B E}$ is always constant when we use FSPM. However, according to (10), the choice of Symbol_Price ${ }_{B E}$, using VSPM, depends on $S_{\max }$.

Fig. 1 shows the price of a reserved symbol using VSPM as a function of $S_{\max }$. We note that Symbol_Price ${ }_{B E}$ increases when $S_{\max }$ increases. According to (10), the price of a reserved symbol is expressed as follows.

$$
p=\frac{\operatorname{Nuser}_{B E}-\frac{n b_{B E}}{S_{\max }}}{\sum_{i=1}^{N u s e r_{B E}} \frac{1}{2 \gamma_{i}}}
$$

Hence, the price of a reserved symbol increases when $S_{\max }$ increases and converges to Nuser $_{B E} / \sum_{i=1}^{N u s e r_{B E}} \frac{1}{2 \gamma_{i}}$.

Fig. 2 shows the mean revenue from the BE connections using VSPM as a function of $S_{\max }$. The mean revenue increases when $S_{\max }$ increases and converges to a constant value. This is because the price actually paid depends on Symbol_Price ${ }_{B E}$ (see (8)).

Fig. 3 shows the mean throughput of the BE connections using VSPM, the rtPS and UGS connections as a function of $S_{\max }$. We notice that the throughput is independent of $S_{\max }$. Indeed, the UGS and rtPS flows being priority over the BE flows, their throughput is not affected by $S_{\max }$. Moreover, for values of $S_{\max }$ above $N_{O F D M}$, (10) always has a positive

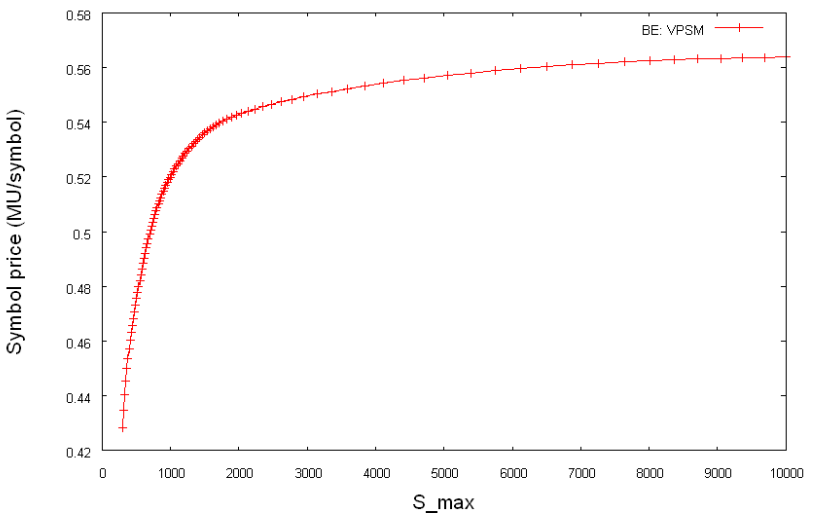

Fig. 1. Symbol_Price ${ }_{B E}$ versus $S_{\max }$

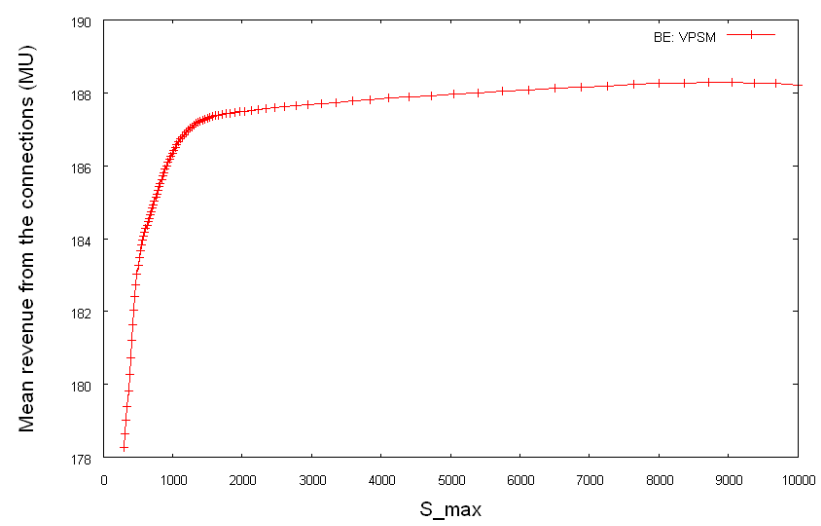

Fig. 2. Mean revenue from BE connections using VSPM versus $S_{\max }$

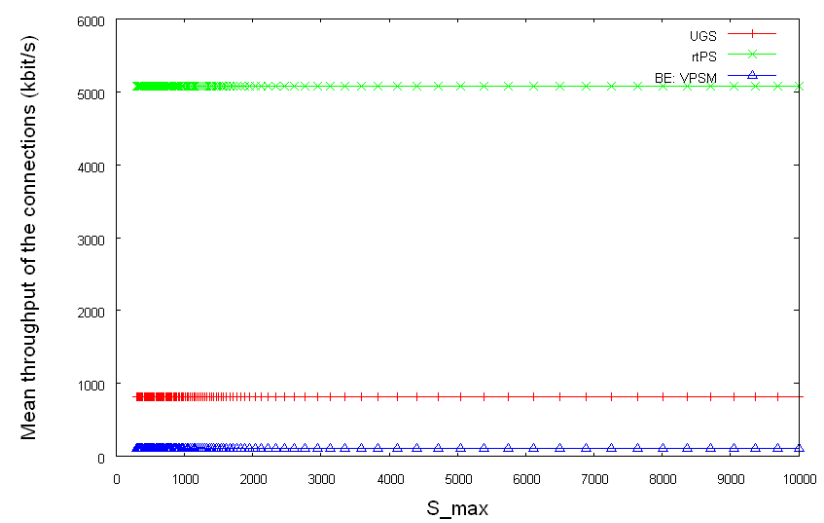

Fig. 3. Mean throughput of BE connections using VSPM versus $S_{\max }$

solution and all the remaining symbols are affected to the BE flows.

\section{Investigation of Symbol_Price ${ }_{B E}$}

In this section, we investigate $S y m b o l_{-}$Price ${ }_{B E}$ when we use FSPM because this parameter is determined using auction in VSPM.

Fig. 4 shows the satisfaction percentage of the BE connections using FSPM as a function of Symbol_Price $B E$. The satisfaction percentage represents the size of all the 


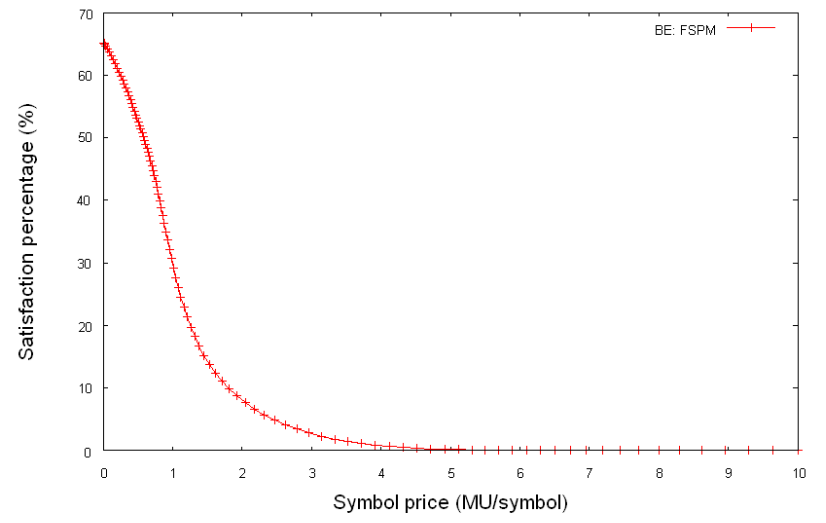

Fig. 4. Satisfaction percentage of BE connections using FSPM versus Symbol_Price $_{B E}$

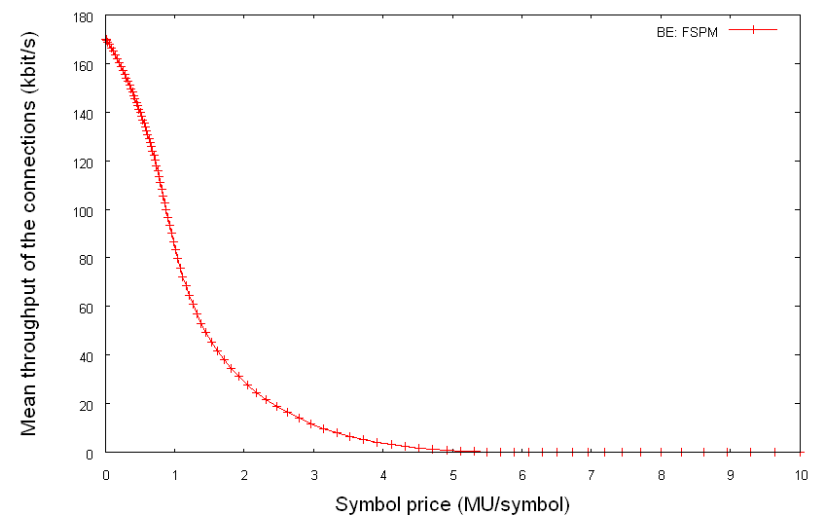

Fig. 5. Mean throughput of BE connections using FSPM versus Symbol_Price $_{B E}$

files already transmitted divided by the total size to transmit. The unsatisfied transfers are due to user refusing the imposed price. We observe that the satisfaction percentage decreases when Symbol_Price ${ }_{B E}$ increases. This is because when the price actually paid increases, it can exceed the valuation function of some $\mathrm{BE}$ users until blocking all the users (Symbol_Price BE $_{\mathrm{B}}>5 \mathrm{MU} /$ symbol).

Fig. 5 shows the mean throughput of the BE connections using FSPM as a function of Symbol_Price ${ }_{B E}$. The mean throughput decreases when Symbol_Price ${ }_{B E}$ increases. This is due to the decrease of the satisfaction percentage.

Fig. 6 shows the mean revenue from the BE connections using FSPM as a function of Symbol_Price $B E$. The revenue from the BE connections increases when Symbol_Price $B E$ increases without exceeding $0.9 \mathrm{MU} / \mathrm{symbol}$. Indeed, despite the decrease of the satisfaction percentage, the price actually paid increases and most of the BE users still afford buying symbols.

When Symbol_Price ${ }_{B E}>0.9 \mathrm{MU} /$ symbol, the revenue from the BE connections decreases when Symbol_Price $B E$ increases. This is because the price actually paid is so high that the majority of the $\mathrm{BE}$ users refuse to reserve symbols. We note that there is no revenue from the $\mathrm{BE}$ connections

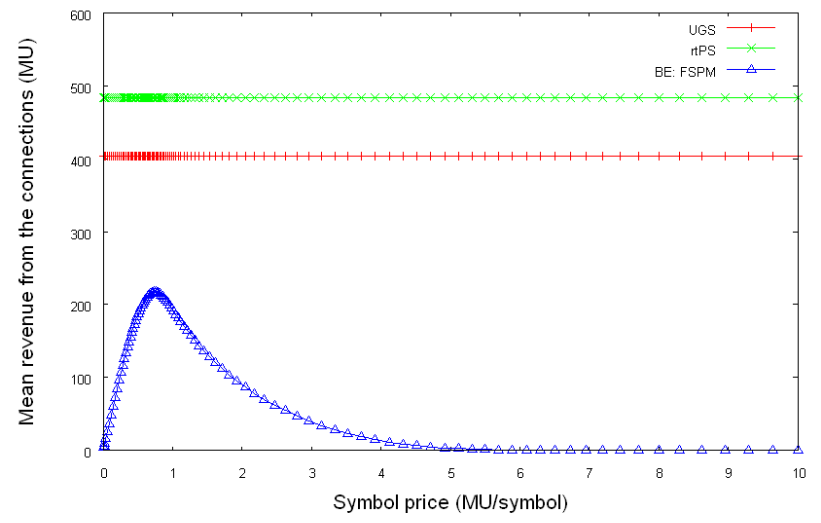

Fig. 6. Mean revenue from BE connections using FSPM versus Symbol_Price $B E$

when Symbol_Price $B E$ exceeds $5 \mathrm{MU} / \mathrm{symbol}$. This is due to the fact that no BE user pay the actual price (see Fig. 4).

We note that FSPM can provide more revenue than VPSM $\left(215 \mathrm{MU}\right.$ when Symbol_Price ${ }_{B E}=0.9 \mathrm{MU} /$ symbol $)$. However, to attain this revenue, the satisfaction percentage decreases from $65 \%$ to $35 \%$ and the mean throughput decreases from $170 \mathrm{kbit} / \mathrm{s}$ to $100 \mathrm{kbit} / \mathrm{s}$. In practice, even if the operator tries to maximize its revenue without taking into account the satisfaction percentage of the BE users, the optimal value of Symbol_Price $B E$ depends on the valuation functions of the BE users. So, the operator has to estimate with accuracy the behavior of the customers because the revenue (and the throughput) can considerably decrease in function of Symbol_Price $B E$.

On the other hand, using VPSM, the operator does not need to determine the symbol price. This pricing model provides a mean revenue larger than $178 \mathrm{MU}$ and a mean throughput equal to $110 \mathrm{kbit} / \mathrm{s}$. In practice, the objective is to maximize the revenue, satisfy the customers and take into account their power of purchase. The best way is to have an auction-based variable price. In our case, the symbol price is between 0.42 $\mathrm{MU} / \mathrm{symbol}$ and $0.58 \mathrm{MU} / \mathrm{symbol}$.

\section{Investigation of $\gamma_{i}$}

In this section, we study the behavior of our pricing models against the valuation parameter $\gamma_{i}$ (in MU by symbol reserved) of the BE users. We consider four continuous uniform distributions on $\left[\Gamma 1_{B E}, \Gamma 2_{B E}\right]:[0.01,0.05],[0.05,0.5],[0.5,0.75]$, and $[0.75,1]$.

Fig. 7 shows the price of a reserved symbol using VSPM as a function of $S_{\max }$. We observe that the mean symbol price increases when $\gamma_{i}$ increases. This is due to the increase of the willingness-to-pay of users (see (7)).

Fig. 8 shows the mean revenue from the $\mathrm{BE}$ connections using VSPM as a function of $S_{\max }$. The revenue from the BE connections increases when $\gamma_{i}$ increases. This is due to the increase of the symbol price. Indeed, the operator must profit from the increase of the valuation functions of the customers.

Fig. 9 shows the mean revenue from the BE connections using FSPM as a function of Symbol_Price ${ }_{B E}$. We note that 


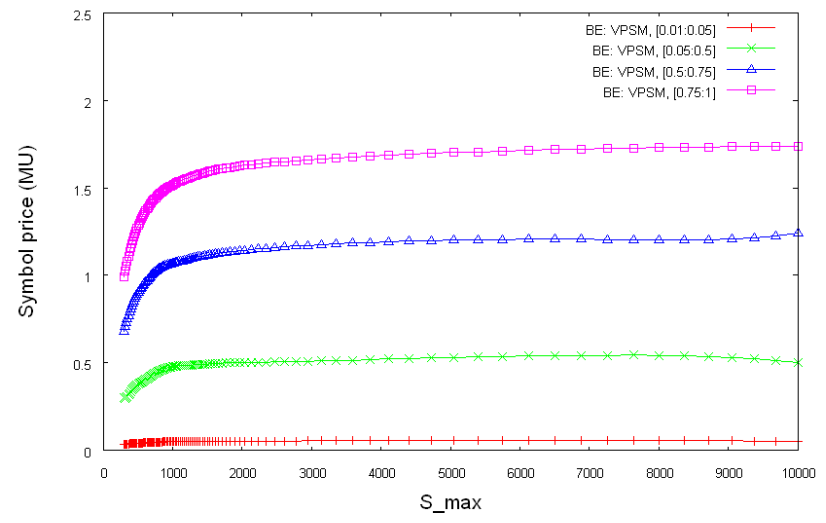

Fig. 7. Symbol_Price ${ }_{B E}$ versus $S_{\max }$

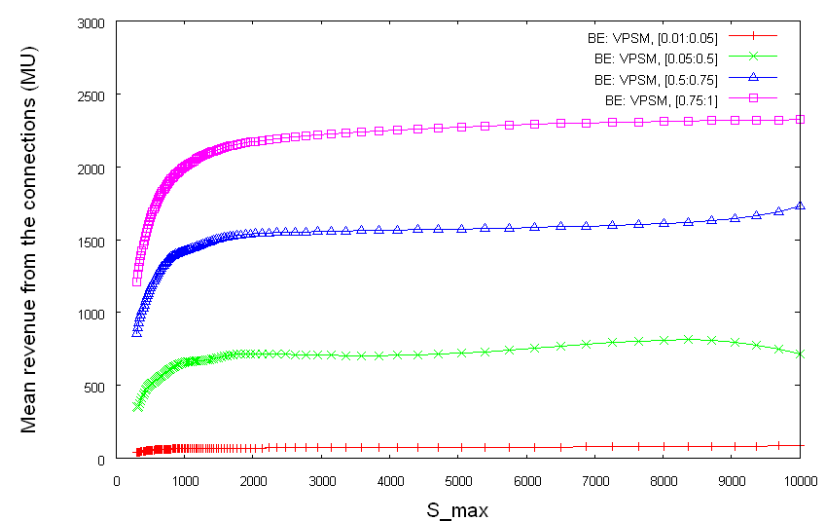

Fig. 8. Mean revenue from BE connections using VSPM versus $S_{\max }$

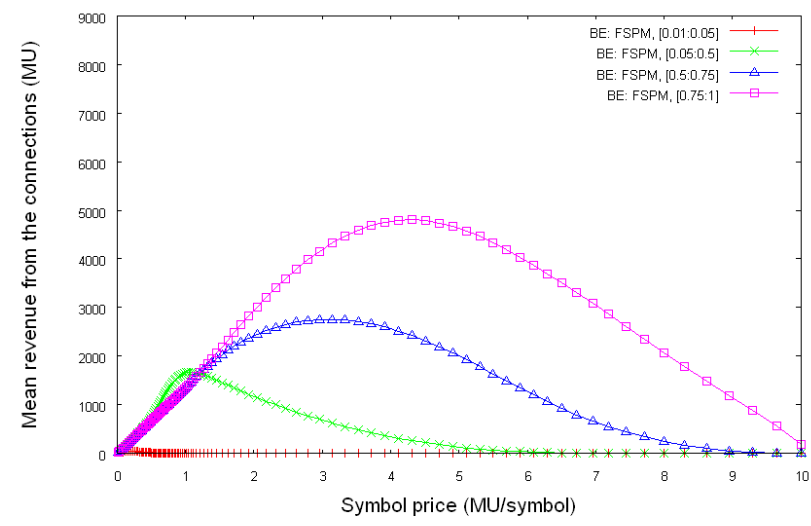

Fig. 9. Mean revenue from BE connections using FSPM versus Symbol_Price $_{B E}$

the symbol price that maximizes the mean revenue increases when $\gamma_{i}$ increases. Symbol_Price $B E$ must be changed from $3 \mathrm{MU} /$ symbol to $4.5 \mathrm{MU} / \mathrm{symbol}$ when $\mathrm{BE}$ customers use $[0.5,0.75]$ instead of $[0.75,1]$. Then, it is recommended to take into account the users behavior dynamically in order to determine the value of symbol Symbol_Price BE $_{\text {maximizing }}$ the operator revenue.

\section{CONCLUSION}

In this paper, we investigated some pricing schemes for a WiMAX system with different classes of QoS. We focus on BE class pricing. We propose two pricing schemes for BE: fixed and variable symbol price (FSPM and VSPM, respectively). The latter is based on auctions. Extensive simulations are conducted to study the behavior of FSPM and VSPM against the different parameters of the pricing models. We showed that FSPM can provide a higher revenue than VSPM. However, FSPM does not take into account the satisfaction of the BE users and the operator have to change the symbol price at each network change. We also investigated the propriety that made VSPM efficient by determining the suitable value of the price symbol that maximize the revenue while distributing all the remaining symbols after serving all the UGS and rtPS customers.

As directions for future work, we can mention the observation of other QoS indicators such as the delay (average or maximum). New pricing optimization considerations should be addressed. The adaptation and application of the proposed schemes to the (Mobile WiMAX) OFDMA Layer is another interesting topic of research as an OFDMA subchannel is a less rigid unit than an OFDM Symbol, especially if we take into account the different types of subchannels defined in IEEE 802.16: full usage of subchannels (FUSC), partial usage of subchannels (PUSC) and Band Adaptive Modulation and Coding (AMC).

\section{REFERENCES}

[1] IEEE 802.16-2004, IEEE Standard for local and metropolitan area networks, Air Interface for Fixed Broadband Wireless Access Systems, Oct 2004.

[2] IEEE 802.16e, IEEE Standard for local and metropolitan area networks, Air Interface for Fixed Broadband Wireless Access Systems, Amendment 2: Physical and Medium Access Control Layers for Combined Fixed and Mobile Operation in Licensed Bands and Corrigendum 1, Feb 2006 (Approved: 7 Dec 2005).

[3] D. Niyato and E. Hossain, "Integration of WiMAX and WiFi: Optimal Pricing for Bandwidth Sharing", IEEE Communications Magazine, vol. 45, no. 5, pp. 140 - 146, 5 May 2007.

[4] "Business Case Models for Fixed Broadband Wireless Access based on WiMAX Technology and the 802.16 Standard", White Paper, WiMAX Forum, 10 October 2004.

[5] J.-W. Lee, M. Chiang, and A. R. Calderbank, "Price-based distributed algorithms for rate-reliability tradeoff in network utility maximization", IEEE Journal on Selected Areas in Communications, vol. 24, no. 5, pp. 962 - 976, May 2006.

[6] B.-J. Chang Y.-L. Chen, and C.-M. Chou, "Adaptive Hierarchical Polling and Cost-based Call Admission Control in IEEE 802.16 WiMAX", Wireless Communications and Networking Conference, 2007, WCNC 2007, pp. 1954 - 1958, Hong Kong, 11-15 March 2007.

[7] A. Belghith, L. Nuaymi, and P. Maille, "Pricing of Real-Time Applications in WiMAX Systems", IEEE 68th Vehicular Technology Conference, VTC2008-Fall, Calgary, Canada, 21-24 September 2008.

[8] D. Besanko and R. R. Braeutigam, Microeconomics, Wiley, January 2005.

[9] X. Wang and H. Schulzrinne, "Auction or tâtonnement - finding congestion prices for adaptive applications", IEEE International Conference on Network Protocols 2002, ICNP 2002, pp. 198 - 199, Paris, France, 12-15 November 2002.

[10] L. Nuaymi, WiMAX: Technology for Broadband Wireless Access, Wiley, January 2007

[11] X. Lagrange, "CIR cumulative distribution in a regular cellular network”, ENST, Tech. Rep., 2000. Available: https://www.rennes.enstbretagne.fr/ xlagrang/Publis/R2000D01-XL.pdf/ 